\title{
DEVELOPMENT OF INDUSTRIAL CONTROLLER FOR SERVO MOTOR IN MECHANICAL SYSTEM
}

\author{
Quang Thinh Truong and Ha Quang Thinh Ngo \\ Graduate Student, Department of Mechatronics Engineering, Faculty of Mechanical \\ Engineering, Ho Chi Minh City University of Technology (HCMUT), 268 Ly Thuong Kiet \\ Street, District 10, Ho Chi Minh City, Vietnam \\ Vietnam National University Ho Chi Minh City, Linh Trung Ward, Thu Duc District, \\ Ho Chi Minh City, Vietnam \\ Thanh Phuong Nguyen* and Hung Nguyen \\ HUTECH Institute of Engineerring, Ho Chi Minh City University of Technology (HUTECH), \\ Ho Chi Minh City, Vietnam \\ *Corresponding Author
}

\begin{abstract}
In this paper, we present the design and implementation of hardware/software for Real-Time Express-based motion controller. This controller can meet the demand for high-speed motion control, high performance which conventional Fieldbus controllers cannot realize. With modular design, the controller brings many benefits such as lowcost, expandable ability, multi-axes control or small physical size. Experimental results for an industrial motion system indicate that the proposed modular controller can perform well in time critical data transmission, feasible and applicable in various fields.
\end{abstract}

Keywords: Motion Control; Modular Design; Real-time Control; Trajectory Planning.

Cite this Article: Quang Thinh Truong, Ha Quang Thinh Ngo and Thanh Phuong Nguyen and Hung Nguyen, Development of Industrial Controller for Servo Motor in Mechanical System. International Journal of Mechanical Engineering and Technology. 11(9), 2020, pp. 1-10. https://iaeme.com/Home/issue/IJMET?Volume=11\&Issue=9

\section{INTRODUCTION}

Motion Control System, which plays as an important role in the modern industry such as Computed Numerically Controlled (CNC) machine tools [1, 2], industrial robots and etc, determines the whole system performance. For the demand for high reliability and high real time manufacturing, modern motion control systems are growing in the direction of high speed, high precision, multi functional and opening that needs embedded and networked 
technique in implement. Networked motion control systems are special networked control systems [3, 4] composed of controllers and multi axes motor drivers, which can ensure the real time synchronization of motion control order and motion state transmission.

A lot of buses based on Ethernet have been introduced into the motion control field, such as PROFIBUS, CAN bus, Interbus-S, SERCOS [5, 6]. C. C. Hsieh and P. L. Hsu [7] proposed a CAN-based motion control system by introducing the event-time structure. Thus, the CANbased motion control system achieves desired control performance even under lower transmission rate or heavier message loading on the bus. B. Chen et al [8] introduced three configurations of the networked synchronized motion control systems. In a networked synchronized motion control system, short communication cycle is needed in order to upgrade the synchronization accuracy. In another approaches, the authors have successfully in developing the Motion Control System with the modern industry Ethernet buses. Y. Hu et al [9] reconfigured the open architecture CNC system based on the Mechatrolink-II Fieldbus [10] that offers interoperability and portability. This component-based CNC architecture is flexible, reconfigurable and more precise performance.

In time critical environment or where complex multi-tasking operations are employed, the execution time of such a software can prove a significant overhead on the system. The development of a real-time executive consisting of both hardware and software co-design methodologies was introduced firstly [11, 12]. The researchers utilized this real-time executive as a prototyping tool for investigating different task scheduling schemes based on high performance evolutionary heuristics. Some of the results obtained by implementing a Hopfield neural network to solve the task scheduling problem in embedded and real-time systems. However, this approach may be not expandable in large scale system and it depends on each application. Related to motion control, the embedded framework and network communication are the cores of multi-axes motion controller. In [13], one kind of modular control component was presented. The network protocol from servo A6N could provide a huge chance to achieve high performance and advanced motion function. Nevertheless, it lacks graphical user interface to assist operator, limited function and big size.

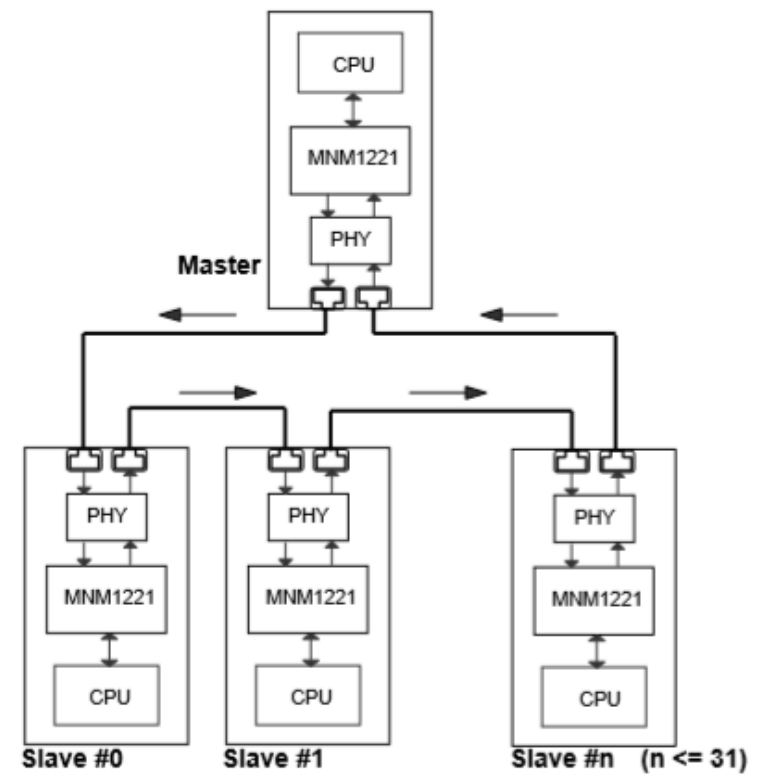

Figure 1. Ring topology of real-time express network.

In this paper, a novel design of network motion module has been developed successfully. Adopting the framework of embedded system, it was integrated into new network communication with highly real-time performance and stable protocol. In this current trend, 
the control issue of multi-axes becomes more critical, especially in large scale system. The problems that were addressed in synchronous control, network topology, time consuming or maintenance service, have been solved by real-time express method. The rest of this paper is organized as follows. Section 2 introduces some definitions, technical specifications and analysis of real-time system. In Section 3, the detailed development of hardware platform and several notifiable remarks are carried out. Section 4 briefly describes the control software which is programmed in $\mathrm{C}++$ and interacted during a cycle servo. The results of experimental module have been complete to validate our proposed approach in Section 5. Several discussions consisting of experiences, practical performance or tested method, are revealed. The conclusions with future work have been demonstrated in Section 6.

Table 1 Combination of period, axes and modes.

\begin{tabular}{|l|l|c|}
\hline Update period & Com. period & Max of axes (16-bit mode) \\
\hline $4 \mathrm{~ms}$ & $2 \mathrm{~ms}$ & 32 \\
\hline $2 \mathrm{~ms}$ & $2 \mathrm{~ms}$ & 32 \\
\hline $2 \mathrm{~ms}$ & $1 \mathrm{~ms}$ & 32 \\
\hline $1 \mathrm{~ms}$ & $1 \mathrm{~ms}$ & 32 \\
\hline $1 \mathrm{~ms}$ & $0.5 \mathrm{~ms}$ & 32 \\
\hline $0.5 \mathrm{~ms}$ & $0.5 \mathrm{~ms}$ & 32 \\
\hline $0.5 \mathrm{~ms}$ & $0.25 \mathrm{~ms}$ & 16 \\
\hline $0.25 \mathrm{~ms}$ & $0.25 \mathrm{~ms}$ & 16 \\
\hline $0.25 \mathrm{~ms}$ & $0.125 \mathrm{~ms}$ & 8 \\
\hline $0.125 \mathrm{~ms}$ & $0.125 \mathrm{~ms}$ & 8 \\
\hline $0.125 \mathrm{~ms}$ & $0.0625 \mathrm{~ms}$ & 4 \\
\hline
\end{tabular}

\section{INTRODUCTION OF REAL-TIME EXPRESS}

Real-time Express is a high-speed synchronous motion network developed by Panasonic Corporation. While being $100 \mathrm{Mbps}$ very high speed (ten times higher than our previous model), the system cost can be kept low by using the commercially available LAN cable. It supports fully synchronization, full-duplex communication and noise immunity with unique error correction. This protocol is connected via ring topology as Fig. 1 with advantages of simple data flow effects, high efficiency and reliability. Comparing with line topology transferring data through many nodes causes low efficiency, there is no cross-talk in ring structure that occasionally is sources of troubles in high-speed data. Such only one master and many slaves, a large scale system could be monitored and managed easily.

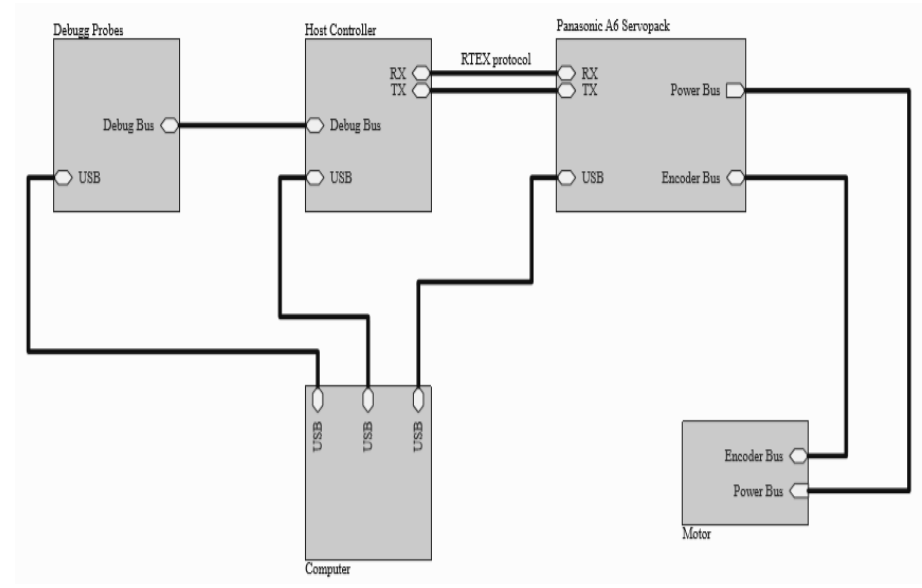

Figure 2. Block diagram of whole master module. 
MNM1221 is a serial interface controller ASIC that enables to establish the real-time communication systems based upon the master-slaves communication. The MNM1221 requires to be used with a PHY (physical layer chip), a pulse transformer and shielded twisted pair cables for 100BASE-TX (IEEE 802.3u) system. In other words, MNM1221 is a special MAC (Media Access Controller) in order to suit 100BASE-TX to real-time communication system for the multi-axis servo control.

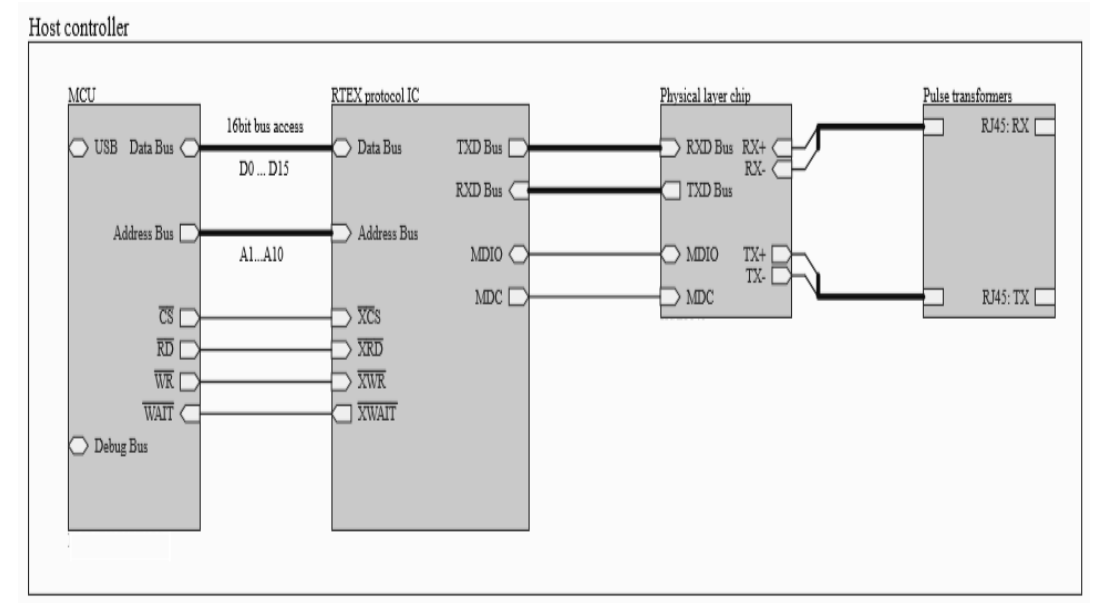

Figure 3. Hardware connection among micro-processor unit, real-time express ASIC chip and peripheral devices.

The MNM1221 serial interface system consists of one master and several slaves, and exchanges the command data from the master and the response data from the slave cyclically. For that, MNM1221 has double banks (buffer) memory for each transmitting and receiving, and this function allows the CPU to operate efficiently.

Table 1 illustrates the information of period, axes and modes. The number of controlled axes depends on different sources such as transferring data mode, communication period and update period. The timing communication is a period of frame transmission while update cycle is to inform data inside the frame. The external device should be limited to join in network since it impacts on number of controlled axes.

\section{DESIGN OF REAL-TIME EXPRESS MODULE}

Traditional Ethernet is not proper to satisfy real-time performance of motion system because of time delay, synchronous transmission or data frame format. To overcome these drawbacks, a module of real-time express communication which are already well-defined to Industrial Ethernet solutions is proposed. Fig. 2 represents the whole linkage in master unit. Once, the operator manipulates on host personal computer in central control room. The popular USB connection assists easy plug-in, fast data transportation between host and network module. There are two selections in circuit schematic, debug for development and release for commerce. The host controller links with servo pack by two LAN wires (receive and transmit) which could extend up to 100 meters. The A6N servo pack-based system is our target of motion controller. To visualize the feed-back signals, a built-in software installed in host computer interconnects with servo via USB type-B.

For the internal bus, the inside hardware schematic is designed in Fig. 3. To expand more controlled axes, 16-byte mode is chosen by connecting BUSMODE pin to ground. The address bus access is from $0 \times 000 \mathrm{~h}$ to $0 \times 200 \mathrm{~h}$ for transmit memory, from $0 \times 200 \mathrm{~h}$ to $0 \times 400 \mathrm{~h}$ for receive memory, from $0 \mathrm{x} 400 \mathrm{~h}$ to $0 \mathrm{x} 480 \mathrm{~h}$ for control registers and the rest for status registers. The timing schedule could be guaranteed owing to CS, RD, WR and WAIT pins. The real-time express protocol IC has two timer sources that are an external timer and an 
internal timer as Fig. 4. For external timer design, it requires that micro-processor provides a tick-clock signal to coordinate the operation of transmit and receive. In the case that using internal timer, MNM1221 utilizes its timer independently. The physical layer chip needs to configure the suitable operating mode from network protocol IC.

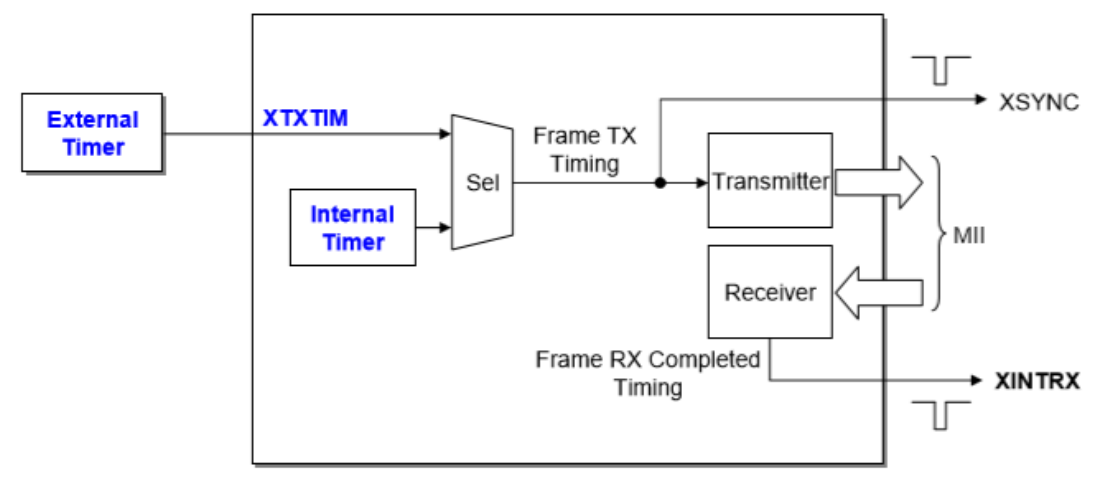

Figure 4. Timer sources of timing frame data.

Communication period, that is the same as transmitting period, can't be set up freely because it must be synchronized with servo control. According to servo specifications, the period must be set to suitable value as accurately as possible. In servo A6N, the communication period must be selected from $2 \mathrm{~ms}, 1 \mathrm{~ms}, 0.5 \mathrm{~ms}, 0.25 \mathrm{~ms}, 0.125 \mathrm{~ms}$ and $0.0625 \mathrm{~ms}$. The command update period is the same or twice as the communication period, and must be selected from $4 \mathrm{~ms}, 2 \mathrm{~ms}, 1 \mathrm{~ms}, 0.5 \mathrm{~ms}, 0.25 \mathrm{~ms}$ and $0.125 \mathrm{~ms}$. The default setting of the servo is that the communication period is $0.5 \mathrm{~ms}$ and the command update period is $1 \mathrm{~ms}$.

\section{DESIGN OF CONTROL SOFTWARE}

In the firmware level, most of operations have been handled by CPU as Fig. 5. Initially, all variables in program should be given default values. Then, several registers of real-time express IC need to set up operating mode such as control registers (M_INIT_DONE, M_INITF_TX, M_TXTIM_SEL), status registers (M_STATE, M_NODE_SUM). The communication in network could be enabled by checking registers (M_TXMEM_SW, M_CYCL_START). There is an infinite loop which often read data from status registers. As a result, the data from numerous slaves is updated frequently.

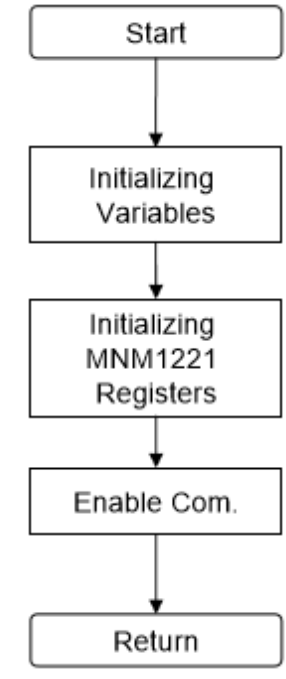

(a)

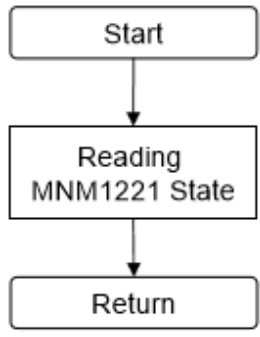

(b)

Figure 5. Flowchart of control firmware, (a) initializing, (b) main loop. 
Since the synchronous interface plays an important role, a routine to incorporate among them is display in Fig. 6. When slave send a request to master, if this message is firstly notified, the readout pointer would record it. To verify the notification message, the process of redundancy check is cyclically executed. If there exists any error in transmission, the counter would label to monitor internal status. Later, this error count is stored in master station. In the case that there is no error, the receive buffer update from network motion IC to internal side. Maybe, user's application takes this information, performs the execution and releases currently internal state to network.

\section{LABORATORY EXPERIMENTS}

To verify the feasible, capable and applicable design in our approach, an experimental test is carried out as Fig. 7. The host PC is Dell Latitude 5500 with powerful Intel core processor. Most of control software would execute in Windows 10 operating system. The information is exchanged between host and motion module thru USB cable. Two LAN wires (from RX port of module to TX port of A6N servo pack and vice versa). One remarkable point is that this module offers on-the-fly adjustment parameters. Hence, as soon as plugging into port, the data flow has been established continuously.

The laboratory validation on one axis has been shown in Fig. 8 and Fig. 9. In S-curve motion profile which is default mode, the command code $(0 \times 10)$ is transmitted from master to servo pack. Together with its, target position and target speed are written in data frame. Optionally, stop mode in byte 3 and absolute/incremental mode in byte 8 are set at start. The experimental position, velocity, acceleration and jerk are illustrated in Fig. 8a, Fig. 8b, Fig. 8c and Fig. 8d respectively. For during one cycle, a master module collects responses from all slaves. In the feed-back frame, except practical data, the servo status consisting of servo ready, alarm, warning message or in position signal aids master to monitor online.

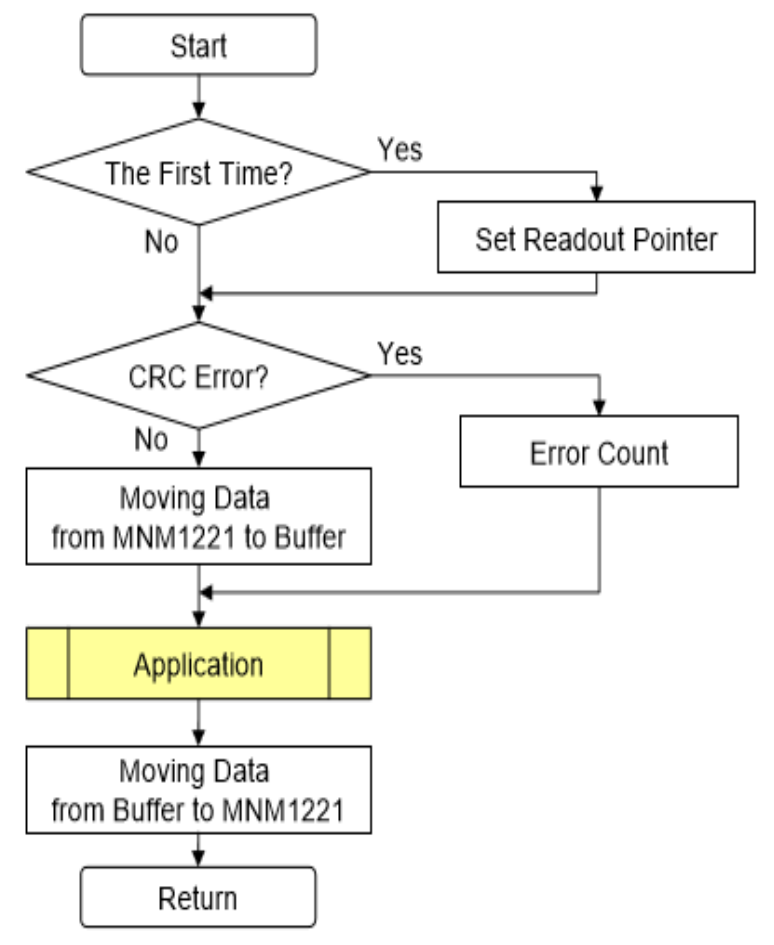

Figure 6. Flowchart of synchronizing routine. 


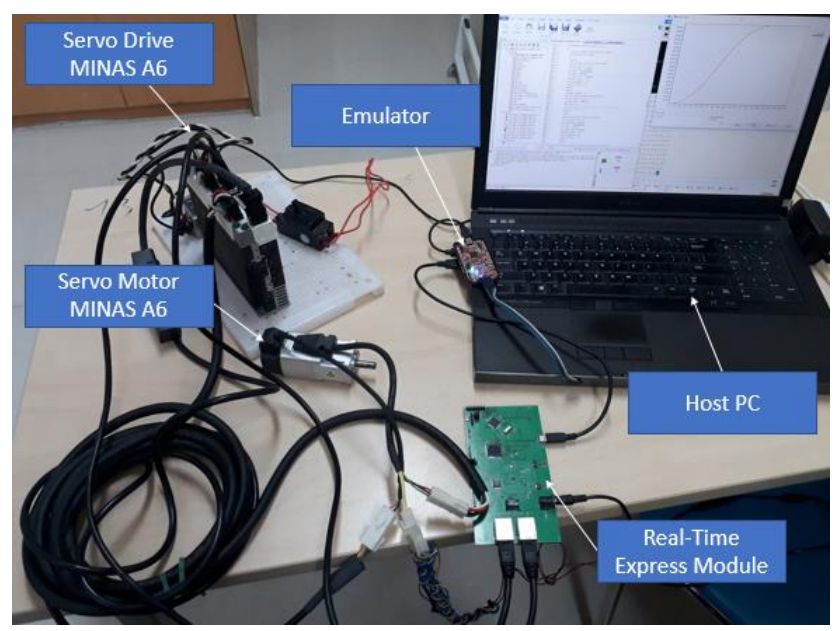

Figure 7. Experimental setup with proposed network module.

For asymmetric S-curve profile from Fig. 8a to Fig. 8d, it involves more setting parameters before executing. The values of acceleration and deceleration are registered in parameter 8.01 and 8.04. Several secondary parameters, for instance digital filter, could bring more clear and enhanced performance. Fig. 8e, Fig. 8f, Fig. 8g and Fig. 8h present actual position, velocity, acceleration and jerk in A6N servo pack.

\section{DISCUSSIONS}

The communication cycle significantly impacts on overall performance of network motion system. It should be considered carefully when designing hardware schematic. This section would analyze and discuss about influence of servo cycle and developing experiences.
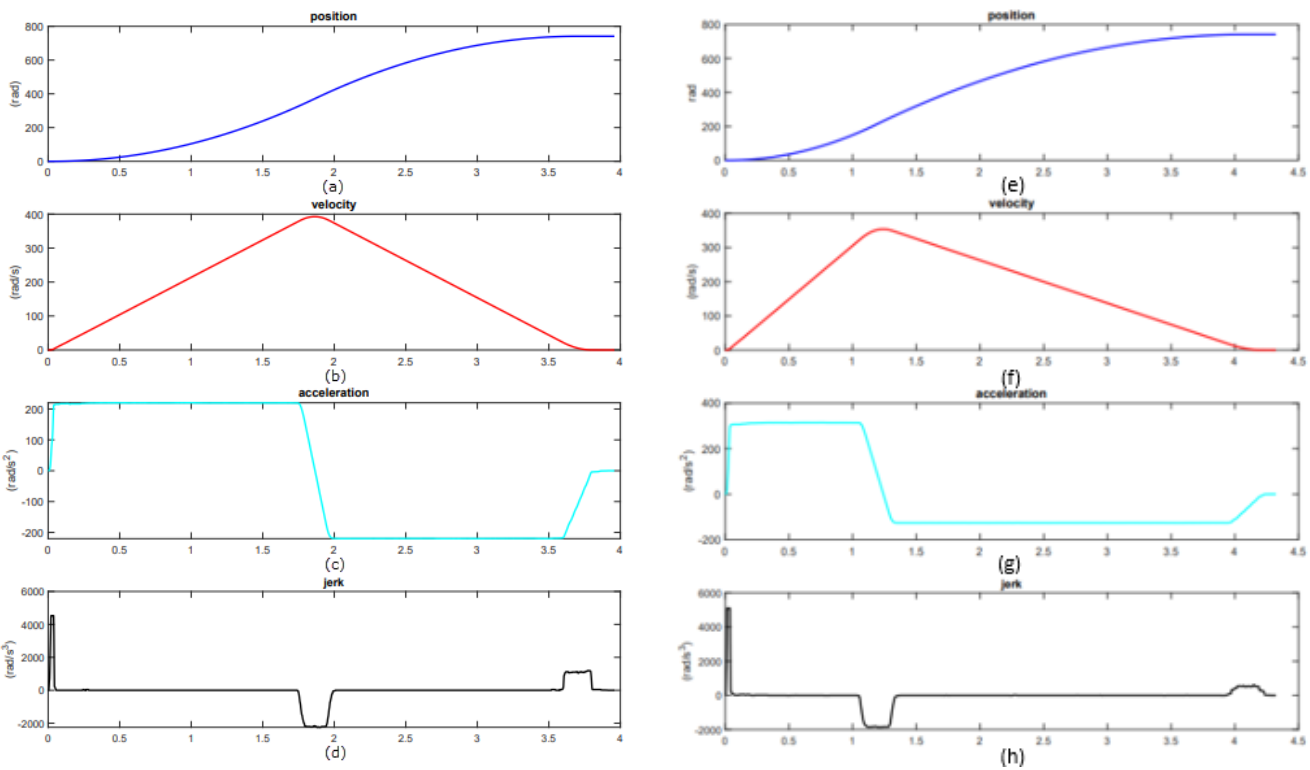

Figure 8. Experimental results of mono-axis control with S-curve profile [left: (a) position, (b) velocity, (c) acceleration, (d) jerk] and AS-curve profile [right: (e) position, (f) velocity, (g) acceleration, (h) jerk].

To control timing in network, the XINTRX, XSYNC and XTXTIM pins handle separated tasks. The XINTRX signal would activate when the procedure of receiving frame is completed. If using an external timing signal to start transmitting the frame in the running state, the cyclical trigger signal must send the acknowledgement signal to XTXTIM pin. In 
this case, the register setting must be needed to switch from the internal timing signal. Reversely, the XSYNC signal is changed logic level when the procedure of transmitting frame starts. In other words, after all slaves receive the data frame in running state, this pin outputs signal in all slaves to synchronize timing pulse. Therefore, it must be ensured that XSYNCs of all slaves are output at the same time as Fig. 9.

General speaking, there are four main states in real-time express protocol IC. The initial state starts firstly to reset registers. In the ring config state, network ASIC chip searches for the information of each slave and configures the operation. The result of this period is to store data into the status registers. After checking the correct slave information by comparing with sampled data, the firmware instructs this chip from ready state to proceed to running state. Otherwise, it returns an error message to notify there is not matched data in network. In running state, using TX and RX memory bank, the firmware is communicated cyclically. The transmission buffer memory (TX memory) and reception buffer memory (RX memory) are composed of two banks respectively. To prevent conflict of data access, one bank is dedicated to the external CPU and another bank is dedicated to the internal communication module. Alternately, the assignment of two banks is switched before reading the received data.

The timing communication during four states is established in Fig. 10. To implement rapidly, the duration of internal timer is equalized to communication period. This timer would provide signal to XTXTIM and guarantee that the low or high width of pulse must keep minimum 1us. In ring config state, a network scan to diagnose slaves which presented in topology should not exceed a period of $2 \mathrm{~ms}$. Once, entering the ready phase, the data transmission has already validated correctly and the registered slave information in master match with up-to-date ones. The transmission speed in running phase is very fast and repeat every cycle. Its speed depends on user's applications and hardware limitation.

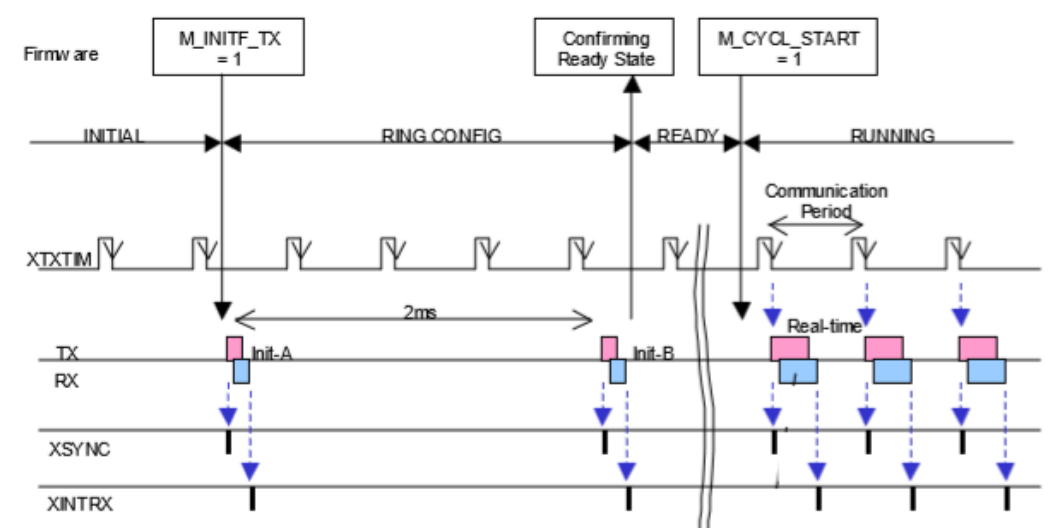

Figure 9. Timing control procedure in transmission protocol.
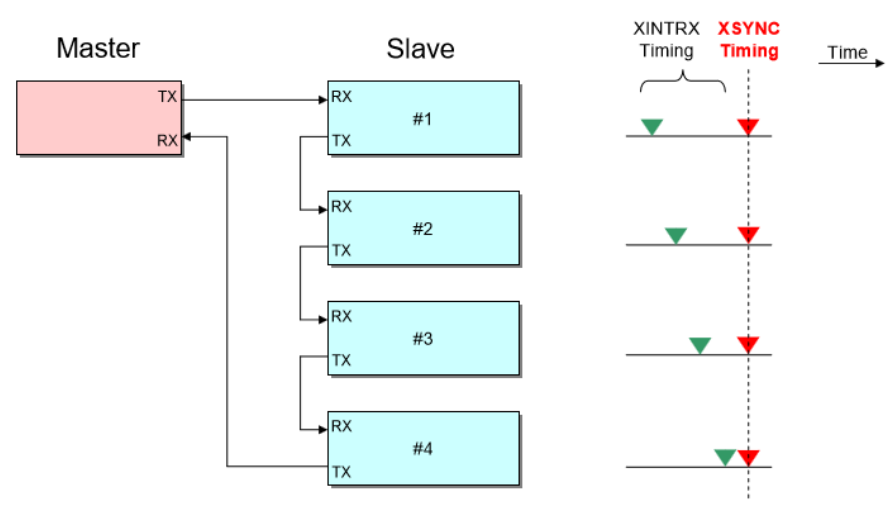

Figure 10. XSYNC timing chart in whole slaves. 
In this paper, a modular design of real-time network controller has been developed successfully. The analyses of network protocol characteristic as well as technical specifications were explained in detail. The testification of circuit schematic and electric connections provide selectable operating mode. In firmware level, the flow of data exchange should be forced to update fluently and continuously. From laboratory experiment, it can be clearly seen that the proposed module can meet the control constraints in motion system, automation factory and robotics system.

\section{ACKNOWLEDGMENTS}

This research is funded by Ho Chi Minh City University of Technology (HCMUT), VNUHCM, under grant number BK-SDH-2020-1870240. We acknowledge the support of time and facilities from Ho Chi Minh City University of Technology (HCMUT), VNU-HCM for this study.

\section{REFERENCES}

[1] Dou, H. (2019). Lyapunov approach for motion synchronization of a two-slider system. Transactions of the Institute of Measurement and Control, 41(14), 4063-4072.

[2] Li, T., Zhang, W. A., \& Yu, L. (2018). Improved Switched System Approach to Networked Control Systems With Time-Varying Delays. IEEE Transactions on Control Systems Technology, 27(6), 2711-2717.

[3] Huang, J., \& Wang, Q. G. (2018). Decentralized adaptive control of interconnected nonlinear systems with unknown control directions. ISA transactions, 74, 60-66.

[4] Xu, X., Gu, G. Y., Xiong, Z., Sheng, X., \& Zhu, X. (2017). Development of a decentralized multi-axis synchronous control approach for real-time networks. ISA transactions, 68, 116126.

[5] Lin, S.Y., Ho, C.Y., Tzou, Y.Y., "Distributed Motion Control Using Real-Time Network Communication Techniques," Proceeding of Third International Power Electronics and Motion Control Conference, vol. 2, pp. 843-847, 2000.

[6] H. Q-. T-. Ngo, T. P-. Nguyen, T. S-. Le, V. N-. S-. Huynh and H. A-. M-. Tran, "Experimental design of PC-based servo system," 2017 International Conference on System Science and Engineering (ICSSE), Ho Chi Minh City, 2017, pp. 733-738.

[7] Hsieh, C.C., Hsu, P.L., "The Event-Time Triggered Network Control Structure for CANbased Motion System," IEEE International Conference on Systems, Man and Cybernetics, pp. 722-726, 2005.

[8] Chen, B., Chen, Y.P., Xie, J.M., Zhou, Z.D., Sa, J.M., "Control Methodologies in Networked Motion Control System," International Conference on Machine Learning and Cybernetics, pp. 1088-1093, 2005.

[9] Hu, Y., Yu, D., Du, S., Zhang, X., Tao, Y., Wang, Z., "Design and Implementation of Reconfigurable CNC System based on Fieldbus," International Conference on Information and Automation, pp. 794-799, 2008.

[10] H. Ngo, Q. Nguyen and W. Kim, "Implementation of input shaping control to reduce residual vibration in industrial network motion system," 2015 15th International Conference on Control, Automation and Systems (ICCAS), Busan, 2015, pp. 1693-1698. 
[11] Seljak, B. K., "Hardware-software co-design for a real-time executive," Proceedings of the IEEE International Symposium on Industrial Electronics, pp. 55-58, 1999.

[12] H. Q. T. Ngo, Q. C. Nguyen and T. P. Nguyen, "Design and implementation of high performance motion controller for 2-D delta robot," 2017 Seventh International Conference on Information Science and Technology (ICIST), Da Nang, 2017, pp. 129-134.

[13] Li, R., Lin, S., Chen, J., "Embedded Motion Controller Design Based on RTEX network," Fifth International Conference on Intelligent Human-Machine Systems and Cybernetics, pp. 326-329, 2013. 